this calculation are shown in the following table. From these results it is seen that the conjecture holds for all primes less than 792,722, which amounts to the first 63,419 primes.

Table of the Function $P(i)$ for $0<i<95$

\begin{tabular}{|c|c|c|c|c|c|c|c|c|}
\hline$i$ & $P(i)$ & $P(i)+i$ & $i$ & $P(i)$ & $P(i)+i$ & $i$ & $P(i)$ & $P(i)+i$ \\
\hline 1 & 3 & 4 & 33 & 867 & 900 & 65 & 23266 & 23331 \\
\hline 2 & 8 & 10 & 34 & 866 & 900 & 66 & 23265 & 23331 \\
\hline 3 & 14 & 17 & 35 & 2180 & 2215 & 67 & 23264 & 23331 \\
\hline 4 & 14 & 18 & 36 & 2179 & 2215 & 68 & 23263 & 23331 \\
\hline 5 & 25 & 30 & 37 & 2178 & 2215 & 69 & 31500 & 31569 \\
\hline 6 & 24 & 30 & 38 & 2177 & 2215 & 70 & 31499 & 31569 \\
\hline 7 & 23 & 30 & 39 & 2771 & 2810 & 71 & 31498 & 31569 \\
\hline 8 & 22 & 30 & 40 & 2770 & 2810 & 72 & 31497 & 31569 \\
\hline 9 & 25 & 34 & 41 & 2769 & 2810 & 73 & 31528 & 31601 \\
\hline 10 & 59 & 69 & 42 & 2768 & 2810 & 74 & 31527 & 31601 \\
\hline 11 & 98 & 109 & 43 & 2767 & 2810 & 75 & 31526 & 31601 \\
\hline 12 & 97 & 109 & 44 & 2766 & 2810 & 76 & 31526 & 31602 \\
\hline 13 & 98 & 111 & 45 & 2765 & 2810 & 77 & 31528 & 31605 \\
\hline 14 & 97 & 111 & 46 & 2764 & 2810 & 78 & 31527 & 31605 \\
\hline 15 & 174 & 189 & 47 & 2763 & 2810 & 79 & 31526 & 31605 \\
\hline 16 & 176 & 192 & 48 & 2763 & 2811 & 80 & 31526 & 31606 \\
\hline 17 & 176 & 193 & 49 & 2763 & 2812 & 81 & 31536 & 31617 \\
\hline 18 & 176 & 194 & 50 & 2763 & 2813 & 82 & 31535 & 31617 \\
\hline 19 & 176 & 195 & 51 & 3366 & 3417 & 83 & 31534 & 31617 \\
\hline 20 & 291 & 311 & 52 & 4208 & 4260 & 84 & 31533 & 31617 \\
\hline 21 & 290 & 311 & 53 & 4207 & 4260 & 85 & 31532 & 31617 \\
\hline 22 & 289 & 311 & 54 & 4206 & 4260 & 86 & 31531 & 31617 \\
\hline 23 & 740 & 763 & 55 & 4205 & 4260 & 87 & 31538 & 31625 \\
\hline 24 & 874 & 898 & 56 & 4204 & 4260 & 88 & 31537 & 31625 \\
\hline 25 & 873 & 898 & 57 & 5943 & 6000 & 89 & 31536 & 31625 \\
\hline 26 & 872 & 898 & 58 & 5944 & 6002 & 90 & 31535 & 31625 \\
\hline 27 & 873 & 900 & 59 & 5943 & 6002 & 91 & 31534 & 31625 \\
\hline 28 & 872 & 900 & 60 & 5942 & 6002 & 92 & 31535 & 31627 \\
\hline 29 & 871 & 900 & 61 & 5941 & 6002 & 93 & 31534 & 31627 \\
\hline 30 & 870 & 900 & 62 & 5940 & 6002 & 94 & 31533 & 31627 \\
\hline 31 & 869 & 900 & 63 & 5940 & 6003 & 95 & $\geq 63324$ & $\geq 63419$ \\
\hline 32 & 868 & 900 & 64 & 5940 & 6004 & 96 & - & - \\
\hline
\end{tabular}

University of California, Los Angeles

\title{
Calculating the Coefficients of Certain Linear Predictors
}

\author{
By D. S. Stoller and L. C. Stoller
}

It is assumed that observations, $x_{j}$, have been made at the $n+1$ points, $j=$ $0,1, \cdots, n$, which are equally spaced. It is desired to find a linear predictor

$$
y_{n+1}=a_{0} x_{0}+\cdots+a_{n} x_{n}
$$

Received 28 September 1958. 
such that $y_{n+1}$ is an estimate of the "next" observation, $x_{n+1}$, which has not yet been made, under the assumptions*

(a) $\sigma_{x_{0}}=\cdots=\sigma_{x_{n}}=\sigma$

(b) $E\left(x_{j}\right)$ is a $k^{\text {th }}$ degree polynomial in $j$

(c) $E\left(y_{n+1}\right)=E\left(x_{n+1}\right)$ for an arbitrary polynomial of degree $k$.

As an ancillary result, Karush and Wolfsohn [1] obtain expressions for the coefficients, $a_{j}$, which minimize $\sigma_{y_{n+1}}^{2} / \sigma^{2}$. These expressions involve determinants of order $k$. Contained herein is an expression for these coefficients which is more convenient for computational purposes. Also a table of some of these $a_{j}$ is deposited in the unpublished mathematical tables repository. (See Reviews and Descriptions of Tables and Books.) By assumption (b) in (2), one can write

$$
E\left(x_{j}\right)=\alpha_{0} P_{0}(j)+\alpha_{1} P_{1}(j)+\cdots+\alpha_{k} P_{k}(j) ; \quad j=0,1, \cdots, n+1
$$

where the $\alpha_{i}$ are constants and where the $P_{i}(j)$ are polynomials in $j$ of degree $i$ which are orthogonal over the set of points, $(0,1, \cdots, n)$, i.e.,

$$
\sum_{j=0}^{n} P_{s}(j) P_{t}(j)=0 \quad s \neq t .
$$

Taking expected values on both sides of equation (1), one can write

$$
E\left(x_{n+1}\right)=a_{0} E\left(x_{0}\right)+\cdots+a_{n} E\left(x_{n}\right)
$$

or, from (3),

$$
\begin{aligned}
& \alpha_{0} P_{0}(n+1)+\cdots+\alpha_{k} P_{k}(n+1) \\
& \quad=a_{0}\left[\alpha_{0} P_{0}(0)+\cdots+\alpha_{k} P_{k}(0)\right]+\cdots+a_{n}\left[\alpha_{0} P_{0}(n)+\cdots+\alpha_{k} P_{k}(n)\right] .
\end{aligned}
$$

From (6) one obtains the following set of equations which the coefficients must satisfy:

Since

$$
\begin{aligned}
& a_{0} P_{0}(0)+\cdots+a_{n} P_{0}(n)=P_{0}(n+1) \\
& a_{0} P_{k}(0)+\cdots+a_{n} P_{k}(n)=P_{k}(n+1) \text {. }
\end{aligned}
$$

it is desired to minimize (8) under the constraints (7). Form the expression

$$
\frac{\sigma_{y_{n+1}}^{2}}{\sigma^{2}}=a_{0}^{2}+\cdots+a_{n}^{2},
$$

$$
\begin{aligned}
F=\left(a_{0}{ }^{2}+\cdots+a_{n}{ }^{2}\right)+\lambda_{0}[ & \left.a_{0} P_{0}(0)+\cdots+a_{n} P_{0}(n)\right] \\
& +\cdots+\lambda_{k}\left[a_{0} P_{k}(0)+\cdots+a_{n} P_{k}(n)\right]
\end{aligned}
$$

where the $\lambda_{i}$ are Lagrange multipliers. Setting $\partial F / \partial a_{j}=0$, one obtains

$$
2 a_{j}+\lambda_{0} P_{0}(j)+\cdots+\lambda_{k} P_{k}(j)=0 \quad j=0,1, \cdots, n .
$$

* The symbol, $\sigma_{x_{i}}$, refers to the standard deviation of $x_{i} ; \sigma$ is a constant; and $E\left(x_{j}\right)$ refers to the expected value of $x_{i}$. 
If these equations are all multiplied by $P_{0}(j)$ and then summed, and this process is repeated but with $P_{1}(j), \cdots, P_{k}(j)$, one obtains the equations (by using (4) and (7)):

$$
\begin{aligned}
& 2 P_{0}(n+1)+\lambda_{0} \sum_{j=0}^{n} P_{0}{ }^{2}(j)=0 \\
& 2 P_{k}(n+1)+\lambda_{k} \sum_{j=0}^{n} P_{k}{ }^{2}(j)=\dot{0} .
\end{aligned}
$$

Therefore, by substituting for the $\lambda_{i}$ in (10), one obtains

$$
a_{j}=\frac{P_{0}(j) P_{0}(n+1)}{\sum_{j=0}^{n} P_{0}{ }^{2}(j)}+\cdots+\frac{P_{k}(j) P_{k}(n+1)}{\sum_{j=0}^{n}{P_{k}}^{2}(j)} ; \quad j=0,1, \cdots, n .
$$

Values for $a_{j}$ were obtained by using the following set of polynomials, which are orthogonal over any set of points symmetric about the origin [2]:

$$
\begin{gathered}
P_{0}(t)=1 \\
P_{1}(t)=t \\
\cdot \\
\cdot \\
\cdot \\
P_{i+1}(t)=t P_{i}(t)-\beta_{i} P_{i-1}(t)
\end{gathered}
$$

where

$$
\beta_{i}=\sum_{j=0}^{n} P_{i}{ }^{2}\left(t_{j}\right) / \sum_{j=0}^{n} P_{i-1}^{2}\left(t_{j}\right)
$$

By transforming the integer set $0,1, \cdots, n$ to the interval $(-2,2)$ we derive $[3]$ a simple expression for the $\beta_{i}$ :

$$
\beta_{i}=\frac{4 i^{2}\left[(n+1)^{2}-i^{2}\right]}{n^{2}\left(4 i^{2}-1\right)}, \quad i=1,2, \cdots, k-1 .
$$

It may be noted that $\sum_{j=0}^{n} a_{j}$ should be equal to one. For degrees one through eight this check sum for the coefficients is verified with a minimum of six significant figures. For degrees nine and ten the check sum is verified to a minimum of five significant figures.

RAND Corporation,

Santa Monica, California and

Space Technology Laboratories,

Los Angeles, California

1. W. KARUSH \& N. Z. WOLFSOHN, "The distance to the origin of a certain point set in $E^{\text {n }}$," Proc. Am. Math. Soc., v. 6, 1955, p. 323-332.

2 GEO. E. FORSYTHE "Generation and use of orthogonal polynomials for data fitting with a digital computer," J. Soc. Indust. Appl. Math., v. 5, 1957.

3. W. E. Milne, Numerical Calculus, Princeton, 1949, p. 265-268. 